\title{
Microwave Kinetic Study of Functional Cellulose Grafted in Homogeneous Media
}

\author{
Gang Chang ${ }^{1, \mathrm{a}}$, Jihua $\mathrm{Li}^{1 . \mathrm{b}}$, Xiaoyi Wei ${ }^{1, \mathrm{c}^{\star}}$, Fei Wang ${ }^{1 \mathrm{~d}}$, Tiaokun $\mathrm{Fu}^{1, \mathrm{e}}$ \\ ${ }^{1}$ Key laboratory of Tropic Crop Products Processing of Ministry of Agriculture, Agriculture Products \\ Processing Research Institute, Chinese Academy of Tropical Agricultural Sciences, Zhanjiang \\ 524001, China \\ achanggang86@126.com, ${ }^{b}$ foodpaper@126.com, ${ }^{c}$ weixywei@163.com, ${ }^{\mathrm{d}}$ rain-wf@163.com, \\ eloveflyfree@126.com
}

Keywords: bagasse cellulose; microwave copolymerization; reaction kinetics

Abstract: The cellulose grafted copolymer was synthesized with 2-(Dimethylamino)ethylmethacrylate(DMAEMA) onto the sugarcane bagasse under microwave irradiation in homogeneous media. A homogeneous solution was obtained through dissolving cellulose in 1-butyl-3-methylinidazolium chloride ionic liquid, which the grafting polymerization initiated by radical polymerizationin, and the presence of $\mathrm{N}_{1} \mathrm{~N}^{\prime}$-methylenebisacrylamide as a crosslinker. During the synthesization, the optimal reaction conditions was completely in a short time, the grating ratio could be close to $100 \%$ in 20 minutes; then the kinetics equation $\mathrm{Rg}=\mathrm{K}[\mathrm{AM}]^{1.4}[\mathrm{AKPS}]^{1.7}$ was established through the process, and the apparent activation energy obtained was $\mathrm{E}=66.373 \mathrm{~kJ} / \mathrm{mol}(\mathrm{R}=0.9915)$ in the initial stage of the reaction. It would provide the basic theory for bagasse cellulose microwave-assisted grafting.

\section{Introduction}

Bagasse is the most abundant natural resources in the tropics areas. It is biodegradable, renewable and added to produce environmentally friendly materials, further itself used as adsorbent [1]. Bagasse mainly contain 46-48\% cellulose, cellulose can be obtained through chemical methods from bagasse and it has a good processability, thermal stability and resistance to water absorption properties. It is necessary to find new technologies to use this renewable macromolecule, which could produce economically valuable products in different processes. Functionality, intelligence and potentials applications which have great significance to achieve a qualitative are the frontier development about the new bio-based materials [2-3].

Natural cellulose has a dense structure with a high degree of crystallinity, so it is difficultly to dissolved in water or organic solvents. However, these methods are environmental pollution, low strength product, low durability, highly cost consumption. The emergence of new solvent system is gradually improved to dissolve cellulose situation, they could well dissolve cellulose and be the reaction medium involved in the reaction. The ionic liquids are easy to dissolve, good solvent, good retention and easy recycling as a new type of green cellulose solvent [4], and it could accelerate the grafting reaction rate as a reaction medium, then cellulose dissolved in ionic liquids can condense out from water, ethanol, acetone and so on, then the remaining solution can be filtered through a rotary evaporator to get reusable ionic liquids[5].

During grafting polymerization, cellulose was carried out by traditional conductive heating. However, it was a comparatively inefficient method and time-consuming for transferring energy into the system. However, microwave irradiation could be directly and uniformly absorbed throughout the entire volume of an object and achieve the remarkable enhancement of the rates over conventional reaction. For instance, Lin [6] et al. reported a homogeneous modification poly Acrylic acid (AA) onto cotton linter by microwave irradiation. They found out that the reaction process was carried out in just three minutes and got a better yield of products. While, grafting kinetics studies were mainly in the traditional way of heating, it was little research on the mechanism of microwave grafted kinetic studies.

In this study, the grafted copolymer(cellulose-g-PDMAEMA) was prepared by grafted functional monomer dimethylaminoethyl methacrylate(DMAEMA) on cellulose surface with the 
ionic liquid phase solution by microwave-assisted heating, which studied the process of reaction conditions, meanwhile the experiment used the estimation method of grafting yield-time curved tangent under different conditions, which obtained a graft copolymerization of the initial polymerization rate $\operatorname{Rg}\left(\mathrm{mol} \cdot \mathrm{L}^{-1} \cdot \mathrm{S}^{-1}\right)$ establishing a process of kinetics equation, that provide a new approach and guidance for bagasse cellulose grafted.

\section{Experimental}

\section{Materials}

Sugarcane bagasse was provided from Guangdong province harvest farm. The bagasse cellulose was dried and collected after washed with deionized water, sodium hydroxide and nitrate. The ionic liquid 1-butyl-3-methlimidazolium chloride $([\mathrm{Bmim}] \mathrm{Cl})$ was synthetized according to the previous work. 2-(Dimethylamino)ethylmethacrylate (DMAEMA, purity $\geq 99.9 \%$ ) was purchased from Sigma. Potassium peroxydisulfate (KPS) was got from Guang hua Chemical Plant. N,N'-methylenebisacrylamide(MBA, purity $\geq 98.0 \%$ ) was obtained from Sinapharm Chemical Reagent Plant. Nitrogen was collected from Zhanjiang oxygen plant. All the other chemicals were of analysis grade, purchased from Guangzhou Chemical Reagent Factory.

\section{Homogeneous synthesis of cellulose-g-PDMAEMA}

The dissolution of cellulose was carried out according to the previous work [7].After completely dissolving the cellulose in 1-butyl-3-methlimidazolium chloride $([\mathrm{Bmim}] \mathrm{Cl})$, the homogeneous solution was placed in a microwave reactor added with a mixed solution of KPS, DMAEMA and MBA $(5 \mathrm{ml})$ in dimethylsulfoxide, and then it was reacted at $70{ }^{\circ} \mathrm{C}$ under nitrogen atomosphere by microwave irradiation $(400 \mathrm{~W} / 50 \mathrm{~Hz})$ in Qpro-M. When the reaction completed, it was added 5 volumes of deionized water to regenerate in a water bath of at $45^{\circ} \mathrm{C}$. After centrifugation, it was washed with ethanol and acetone solution at $57^{\circ} \mathrm{C}$ soxhlet reflux for $24 \mathrm{~h}$. The precipitate was then centrifuged and dried.

\section{Kinetic studying of microwave graft homogeneous}

In this experiment, the reaction could achieve high grafting rates with microwave-assisted in the relatively short period of time, the reaction could be approximately considered a constant rate in a short reaction time, which show an approximately straight line about the grafting rate in a short reaction time. The test used the estimation method of grafting yield-time curved tangent under different conditions, which obtained a graft copolymerization of the initial polymerization rate $\operatorname{Rg}\left(\mathrm{mol} \cdot \mathrm{L}^{-1} \cdot \mathrm{S}^{-1}\right)$. Calculated was as follows (Eq.1):

$$
\mathrm{Rg}=[\mathrm{Mo}] \frac{\mathrm{dC}}{\mathrm{dt}}
$$

Where Mo is the initial molar concentration of monomer $\left(\mathrm{mol} \cdot \mathrm{L}^{-1}\right), \mathrm{C}$ is the grafting rate ratio.

\section{Results and Discussion}

The fig. 1 showed the different grafted rate with different reaction time. The grafting rate showed a gradual increasing trend with the growth of time. Firstly the graft polymerization rate was not fast due to less number of free radicals generated by oxidation of KPS as the reaction started. then a sufficient number of free radicals were generated with time increased, which accelerated the radicals active contact between monomer and cellulose macromolecules, thereby the grafting rate increased accordingly with the reaction rate of the graft polymerization. 


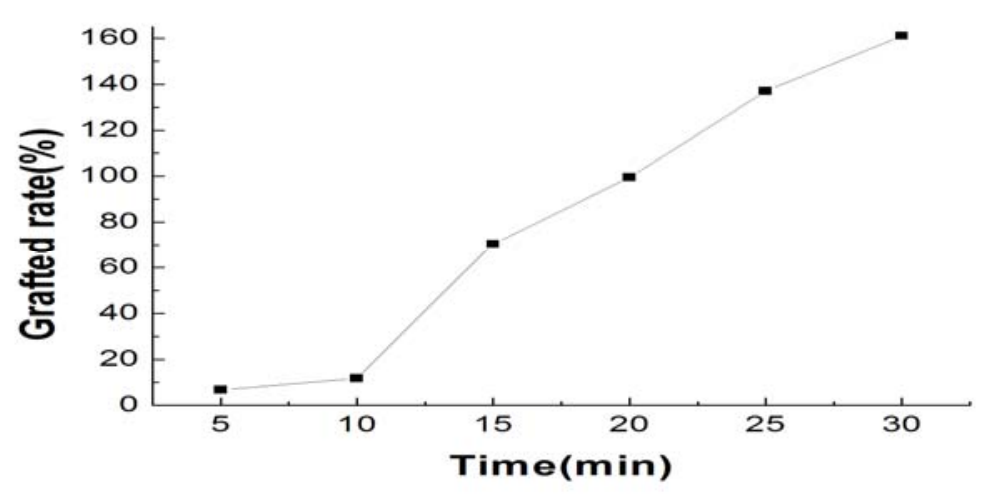

Fig.1 The influence of reaction time on grafting

Bagasse cellulose amount $0.5 \mathrm{~g}$, monomer content was $1: 3,1: 4,1: 5$, the reaction temperature was $75^{\circ} \mathrm{C}$, KPS concentration investigated was $2.4 \%$ within the graft ratio at different times. And then calculate the reaction rate $\mathrm{Rg}$ according to the Eq.1, The results are shown in table 1:

Table 1 Results of analysis process for amount of monomer to grafted rate

\begin{tabular}{lccccr}
\hline Monomer content Time $(\min )$ & $\mathrm{C}(\%)$ & $\operatorname{Rg}\left(\mathrm{mol} \cdot \mathrm{L}^{-1} \cdot \mathrm{s}^{-1}\right)$ & $\ln R g$ & $\ln [\mathrm{AM}]$ \\
\hline \multirow{4}{*}{$1: 3$} & 10 & 1.66 & & & \\
& 15 & 50.48 & 1.45 & 0.3715 & -1.5141 \\
20 & 75.82 & & & \\
& 25 & 103 & & & \\
$1: 4$ & 10 & 6.76 & & & \\
& 15 & 55.65 & 2.10 & 0.7419 & -1.2730 \\
& 20 & 90.47 & & & \\
& 25 & 120 & & & \\
$1: 5$ & 10 & 11.76 & & & \\
& 15 & 70.48 & 2.91 & & \\
& 20 & 99.55 & & & \\
& 25 & 137 & & & \\
\hline
\end{tabular}

Then plotting $\ln \operatorname{Rg}$ and $\ln [\mathrm{AM}]+2$ obtained, the relationship equation about the monomer amount and the graft rate was $\mathrm{y}=1.4137 \mathrm{x}-0.3053\left(\mathrm{R}^{2}=0.9988\right)$, it showed reaction order 1.4 corresponding to the amount of monomer, that $\operatorname{Rg} \propto[\mathrm{AM}]^{1.4}$.

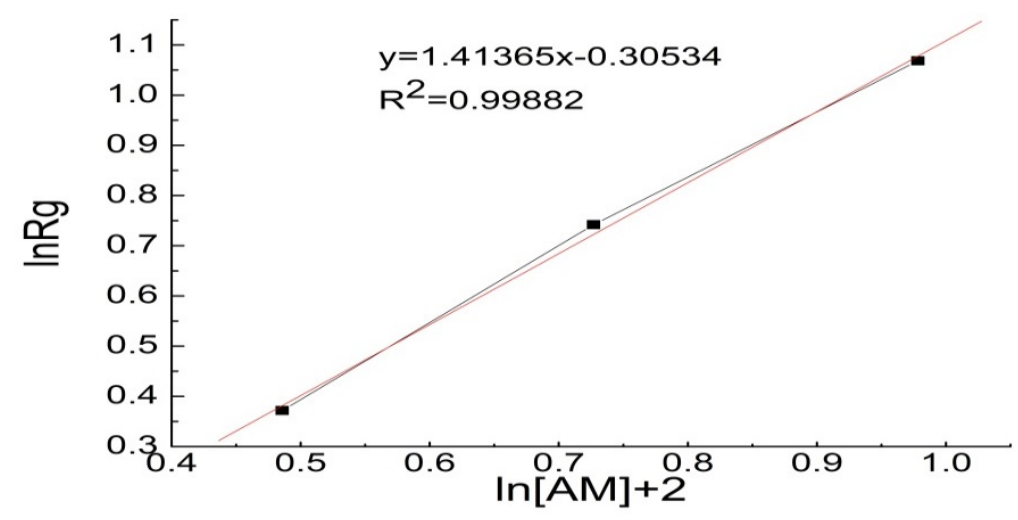

Fig.2 The linear regression of monomer

Bagasse cellulose amount $0.5 \mathrm{~g}$, monomer content was $1: 5$, the reaction temperature was $75^{\circ} \mathrm{C}$, KPS concentration investigated was $1.4 \%, 2 \%$ and $2.4 \%$ within the graft ratio at different times, 
And then calculate the reaction rate $\mathrm{Rg}$ according to the Eq.1.The results are shown in table 2:

Table 2 The results of analysis process of the concentration of initiator to grafted rate

\begin{tabular}{|c|c|c|c|c|c|c|}
\hline KPS( $(\%)$ & Time (min) & $\mathrm{C}(\%)$ & & $\left.{ }^{-1} \cdot \mathrm{s}^{-1}\right)$ & $\operatorname{lnRg}$ & [PS ] \\
\hline & 10 & & 4.71 & & & \\
\hline 1.4 & 15 & & 28.20 & 1.17 & 0.1570 & -5.9522 \\
\hline & 20 & & 39.82 & & & \\
\hline & 25 & & 55.00 & & & \\
\hline & 10 & & 6.25 & & & \\
\hline 2.0 & 15 & & 37.35 & 2.12 & 0.7514 & -5.5994 \\
\hline & 20 & & 73.09 & & & \\
\hline & 25 & & 92.61 & & & \\
\hline & 10 & & 11.76 & & & \\
\hline 2.4 & 15 & & 70.48 & 2.91 & 1.0682 & -5.4262 \\
\hline & 20 & & 99.55 & & & \\
\hline & 25 & & 137 & & & \\
\hline
\end{tabular}

Then plotting $\ln R g$ and $\ln [\mathrm{AKPS}]+6$ obtained, the relationship equation about the KPS concentration and the graft rate was $y=1.725 x+0.0711\left(R^{2}=0.9998\right)$,it showed reaction order 1.7 corresponding to the initiator concentration, that $\operatorname{Rg} \propto[\mathrm{AKPS}]^{1.7}$.

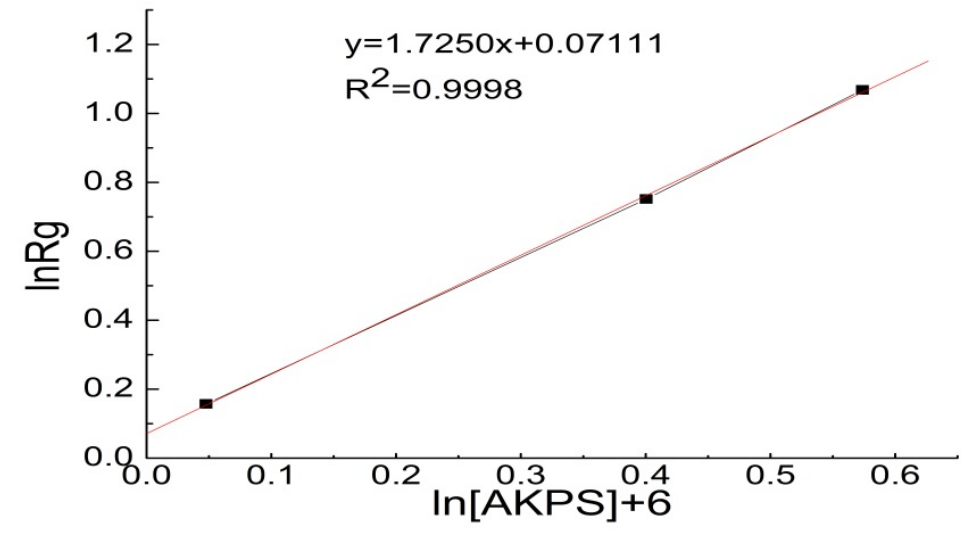

Fig.3 The linear regression of initiator

Bagasse cellulose amount $0.5 \mathrm{~g}$, monomer content was $1: 5$, KPS concentration investigated was $2.4 \%$, the reaction temperature was $55^{\circ} \mathrm{C}, 65^{\circ} \mathrm{C}$ and $75^{\circ} \mathrm{C}$ within the graft ratio at different times. And then calculate the reaction rate $\mathrm{Rg}$ according to the Eq.1. The results are shown in table 3:

Table 3 The results of analysis process of the reaction temperature to graft rate

\begin{tabular}{|c|c|c|c|c|c|}
\hline \multicolumn{2}{|c|}{ Temperature $\left({ }^{\circ} \mathrm{C}\right) \operatorname{Time}(\min )$} & $\mathrm{C}(\%)$ & $\operatorname{Rg}\left(\mathrm{mol} \cdot \mathrm{L}^{-1} \cdot \mathrm{s}^{-1}\right)$ & \multicolumn{2}{|l|}{$\ln R g$} \\
\hline \multirow{4}{*}{55} & 10 & 7.69 & & \multirow{2}{*}{\multicolumn{2}{|c|}{$-1.07880 .01818$}} \\
\hline & 15 & 12.68 & 0.34 & & \\
\hline & 20 & 17.01 & & & \\
\hline & 25 & 22.08 & & & \\
\hline \multirow{3}{*}{65} & 10 & 12.48 & & & \\
\hline & 15 & 17.21 & 0.99 & -0.009 & 0.01538 \\
\hline & 20 & 22.37 & & & \\
\hline \multirow{5}{*}{75} & 25 & 56.64 & & & \\
\hline & 10 & 11.76 & & & \\
\hline & 15 & 70.48 & 2.91 & 1.0682 & 0.01333 \\
\hline & 20 & 99.55 & & & \\
\hline & 25 & 137 & & & \\
\hline
\end{tabular}


Then plotting $\ln \operatorname{Rg}+1$ and $(1 / \mathrm{T}) \times 10^{2}$ obtained, the relationship equation about the reaction temperature and the graft rate was $y=-4.0166 x+7.3254\left(\mathrm{R}^{2}=0.9832\right)$. Meanwhile both sides of the equation logarithmic according to Arrhenius equation as follows, the apparent activation energy obtained was $\mathrm{E}=66.373 \mathrm{~kJ} / \mathrm{mol}(\mathrm{R}=0.9915)$ in the initial stage of the reaction. $\ln \mathrm{Rg}=\ln \mathrm{K}-\mathrm{E} / \mathrm{RT}$

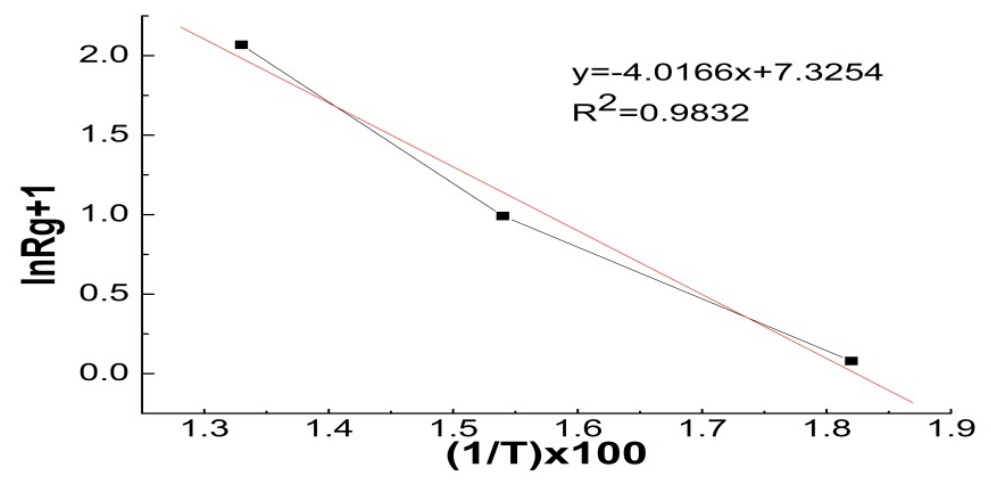

Fig.4 The linear regression of temperature

\section{Conclusions}

The grafted copolymer cellulose-g-PDMAEMA was prepared by microwave-assisted in a homogeneous system. By the research of monomer amount, initiator agent and temperature on grafting reaction rate, the equation of graft copolymerization reaction rate obtained under microwave: $\mathrm{Rg}=\mathrm{K}[\mathrm{AM}]^{1.4}[\mathrm{AKPS}]^{1.7}$, the apparent activation energy obtained was $\mathrm{E}=66.373 \mathrm{~kJ} / \mathrm{mol}$ $(\mathrm{R}=0.9915)$ in the initial stage of the reaction, which provided basic kinetics research about microwave graft reaction, and the work is further in process.

\section{Acknowledgements}

We gratefully acknowledge the Fundamental Scientific Research Funds for Chinese Academy of Tropical Agricultural Sciences (No.1630062013012), The work is also supported by the same funding related projects ((No.1630062015016) and (No.1630062015020).

\section{References}

[1] KX Li, KL Huang: Popular Science Vol. 2(2011),P.91.

[2] E Bianchi, A Bonazza, E Marsano and S Russo: Carbohydrate Polymers Vol. 41 (2004), P.47.

[3] CF Liu, RC Sun, AP Zhang, JL Ren, XA Wang, MH Qin, ZN Chao and W Luo: Carbohydrate Research Vol.342(2007),P.920.

[4] B Philipp, W Wagenknecht: Cellulose Chemistry and Technology Vol.17(1983), P446..

[5] C Iee.,US, Patent 6,129,867, (2000)

[6] C.X. Lin, H.Y. Zhan, M.H. Liu, S.Y. Fu and L.H. Huang: Appl. Polym. Sci. Vol.118 (2010), p.399.

[7] J.H. Li, Y.F. Tang, J.C. Chen, Y.P. Cao, M.F. Huang, T.K. Fu and J.B. Su: Adv. Mater. Res. Vol.152-153 (2011), p. 1570. 